\section{A Prospective, Comparative Trial to Optimize Sampling Techniques in EUS- Guided FNA of Solid Pancreatic Masses}

\author{
Lee JK, Choi JH, Lee KH, Kim KM, Shin JU, Lee \\ JK, Lee KT, Jang KT \\ Gastrointest Endosc 2013;77:745-51
}

Endoscopic ultrasound guided fine needle aspiration (EUS-FNA) is a preferred diagnostic modality for evaluation of solid pancreatic lesions as it is safe and has high diagnostic accuracy. ${ }^{1,2}$ The diagnostic yield of EUS-FNA depends on number of variables that include the size of the lesion, experience of the endosonologist, the guage of the needle, the number of passes, presence of onsite cytopathologist, methods used for slide preparation and the location of the mass in the pancreas. ${ }^{3,4}$ There have been numerous efforts aiming to increase the tissue yield and improve the sensitivity of diagnosis of pancreatic masses without increasing the complication rates. However, because of lack of evidence there is debate on the use of suction during EUS FNA and also there is no uniformity on the method of expressing the material on the slides after FNA i.e., use either the method of reinserting the stylet or using the air flushing method). To answer these questions the authors of the current study conducted a prospective, comparative trial to compare the sample quality and diagnostic yield between samples with suction $(\mathrm{S}+)$ versus no suction (S-) during EUS FNA and also samples expressed by reinserting the stylet (RS) versus by air flushing (AF).

In this study 81 consecutive patients underwent EUSFNA with a 22 or 25 gauge needle (Endocoil with 22-gauge and Echotip with 25-gauge; Cook endoscopy, WinstonSalem, NC) and the same needle was used for making four passes into the lesion with the needle being passed to and fro inside the lesion for 10 times during each pass. During the procedure, a ten $\mathrm{ml}$ syringe was attached to the hub for aspiration using suction whilst no syringe was attached for puncturing without suction. Also once a pass had been taken the expression of contents was done either by slow reinsertion of stylet or by controlled application of air by a 10 $\mathrm{ml}$ syringe. Therefore, four different combinations of methods were used: No suction and expression by stylet, no suction and expression by air pressure, aspiration by suction and expression by stylet or aspiration by suction and expression by air pressure. Since each lesion underwent four passes, each pass was randomly assigned to the four groups. Smears were made by the endosonographers and fixed in absolute alcohol and a total of 324 aspirations were done in 81 patients.

There was one false positive and four false negative aspirations in all. On comparing the samples taken $\mathrm{S}+$ group with S-group the authors found that the diagnostic yield was significantly higher with the use of suction $(72.8 \%$ versus $58.6, p=0.001$ ) with the use of suction being associated with higher cellularity. But, the use of suction was also associated with a higher blood contamination and no air drying artifacts were observed in either group. When the comparison of method of deliver of sample was made, the diagnostic samples were similar whether samples were delivered using reinsertion of stylet or by use of air flushing $(66.7 \%$ and $64.8 \%, p=0.608$ ). However, the blood contamination was found to be more with the use of stylet. Further, the authors report that use of suction and the technique of expression technique work independent of each other.

\section{Commentary}

This report is a well-planned study to resolve the dilemma whether the application of suction and use of air flushing affect the quality of samples achieved by EUS-FNA. The authors have concluded that the use of suction during puncturing the lesion and expression of the material by air flushing may be used preferentially in pancreatic EUS-FNA as they were more effective and convenient techniques. Better yield with suction may be possible because of the possibility of the tissue being held against the cutting edge of the needle by the aspirating pressure. However, earlier studies on the issue of using suction during FNA have yielded conflicting results with many studies suggesting that suction did not improve the diagnostic yield and also increased the blood contamination of the specimen. ${ }^{6-8}$ But majority of these studies had FNA being done from variety of lesions and these results may not be applicable in patients of pancreatic cancer, the tumors that have low cellularity and increased fibrosis. Apart from resolving this issue of using suction during FNA of pancreatic solid lesions, the authors have also addressed an important issue of expressing the material from the needle, an issue that has not been systematically addressed so far.

We, at our centre, utilise the suction method and express the material by air flushing and found an excellent diagnostic accuracy. Sahai et al in their study of 135 solid masses expressed the samples by air flushing and suggested that the clotting in the needle was rare if aspirated material 
was expelled immediately without delay. ${ }^{9}$ However, use of air flushing must be controlled as inappropriate pressure may result in spraying of the material. However, single centre study and use of needles of different gauge size are limitations of the current study. It also would be interesting to note differences, if any, between the diagnostic yield of first pass and subsequent passes as the increasing number of passes makes the needle deformed and blunted.

\section{Randomized Trial Comparing Fanning with Standard Technique for Endoscopic Ultrasound-guided fine- needle Aspiration of Solid Pancreatic Mass Lesions}

\author{
Bang JY, Magee SH, Ramesh J, Trevino JM, \\ Varadarajulu S \\ Endoscopy 2013 early online; doi 10.1055/s-0032-1326268
}

Endoscopic ultrasound guided fine needle aspiration (EUS-FNA) is a preferred diagnostic modality for evaluation of solid pancreatic lesions as it is safe and has high diagnostic accuracy. ${ }^{1,2}$ As mentioned earlier, the diagnostic yield of EUSFNA depends on number of variables that include the size of the lesion, experience of the endosonologist, the guage of the needle, the number of passes, presence of onsite cytopathologist, methods used for slide preparation and the location of the mass in the pancreas. ${ }^{3,4}$ Certain technical manoeuvres like doing sampling from the lesion's edge and the use of "fanning" have been described to increase the diagnostic yield. However, these techniques have not been compared with the standard FNA techniques. In the current study, the authors conducted a randomized trial comparing the fanning technique with the standard approach where only a single area within the mass was targeted and tried to determine if fanning could enhance diagnostic accuracy and whether the number of passes needed to achieve a diagnosis could be reduced.

The trial was done by using a single needle (25 Gauge for head and uncinate and 22 Gauge for lesions of body and tail; Expect; Boston Scientific Corp., Natick, Massachusetts, USA) and a single expert endosonologist did all the procedures. In the standard technique the tip of the needle was positioned at a single location and moved 16 times to target the periphery of the lesion. In the fanning technique the needle was positioned at four different areas within the mass and moved back and forth four times. On site pathologist, blinded to the EUS-FNA technique, immediately interpreted the yield thereby allowing for assessment of number of passes. A maximum number of three passes were allowed per technique and if required the patient was crossed over to the second technique. Of the 54 patients eventually randomised, 26 were randomised to standard EUS-FNA and 28 were randomised to the fanning technique. Although the two groups were comparable in tumour characteristics, tumour location, or the route of FNA but the fanning group had lesions with a smaller size than the standardized group ( $30 \mathrm{~mm}$ versus $40 \mathrm{~mm} ; p=0.009$ ). The total number of passes needed to achieve a diagnosis was significantly lesser in the fanning group $(p=0.02)$. Also a higher proportion of patients achieved a diagnosis on first pass in the fanning technique ( $85.7 \%$ vs. $57.7 \% ; p=0.02$, respectively). The diagnostic accuracy was comparable for the two groups (Fanning 96.4\% and Standard $76.9 \% ; p=0.05$ ). A higher number of patients in the standard technique needed to be crossed over to the fanning technique (six versus one). No procedural complications or needle dysfunction was encountered in either group. The authors concluded that the fanning technique of FNA is superior to the standard approach as fewer passes are required to establish a diagnosis.

\section{Commentary}

This is a well conducted randomized study that has established the superiority of the fanning technique for EUS FNA. One earlier report had also indicted that EUS-FNA from the multiple sites might increase the yield form pancreatic masses. ${ }^{10}$ However, it would be interesting to know the sub-analysis of small lesions as fanning may be difficult in very small lesions. The current study included only solid lesions of the pancreas and the results if the fanning technique needs to be addressed for other lesions and results from other centres with varying underlying lesions are awaited before finally it can be said that fanning technique is the best. Summing up the two studies discussed in this section, it appears the EUS guided FNA being done with suction and using fanning technique with the sample being expressed using air suction may be the preferred method of doing EUS FNA for solid pancreatic lesions.

\section{References}

1. Hewitt MJ, McPhail MJ, Possamai L, Dhar A, Vlavianos P, Monahan KJ. EUS-guided FNA for diagnosis of solid pancreatic neoplasms: a meta-analysis. Gastrointest Endosc 2012;75:319-31.

2. Rana SS, Bhasin DK, Srinivasan R, Sampath S, Mittal BR, Singh K. Distinctive endoscopic ultrasound features of isolated pancreatic tuberculosis and requirements for biliary stenting. Clin Gastroenterol Hepatol 2012; 10:323-325.

3. Siddiqui AA, Brown LJ, Hong SK, Draganova-Tacheva RA, Korenblit J, Loren DE. Relationship of pancreatic mass size and diagnostic yield of endoscopic ultrasound-guided fine needle aspiration. Dig Dis Sci 2011; 56:3370-5.

4. Savides TJ. Tricks for improving EUS-FNA accuracy and maximizing cellular yield. Gastrointest Endosc 2009;69: S130-3.

5. Thomson HD. Thin needle aspiration biopsy. Acta Cytol 1982; 26:262-3 